\title{
EVALUACIÓN DE DOS COMPLEJOS ENZIMÁTICOS FIBROLÍTICOS COMERCIALES SOBRE LA DIGESTIBILIDAD Y LA CINÉTICA DE DIGESTIÓN DEL COGOLLO DE CAÑA DE AZÚCAR (Saccharum officinarum)
}

\author{
Evaluation of Two Enzymatic Fibrolytic Commercial Complexes on \\ Digestibility and Digestion Kinetics of Sugarcane Bud \\ (Saccharum officinarum)
}

\author{
José Delgado S. ${ }^{1}$, Juan Olazábal L. ${ }^{1}$, Fernando Carcelén C. ${ }^{1}$, Teresa Arbaiza F. ${ }^{1}$, \\ Miguel Ara G. ${ }^{1}$, Karina Bardales O. ${ }^{1}$, Felipe San Martín H. ${ }^{1,2}$
}

\section{Resumen}

Se evaluó el efecto de dos complejos enzimáticos comerciales (Allzyme Vegpro® y Rovabio $^{\mathrm{TM}}$ Excel) sobre la digestibilidad in situ y la cinética de digestión de la materia seca del cogollo de caña de azúcar (Saccharum officinarum). El sustrato fue secado, molido, pasado por un tamiz de $3 \mathrm{~mm}$ y tratado con las enzimas $30 \mathrm{~min}$ antes de la incubación. La concentración del complejo enzimático fue del $1 \%$ respecto al sustrato. Además, se usó sustrato sin ser tratado como tratamiento control. El diseño experimental fue de bloques al azar con cuatro periodos, tres tratamientos y dos repeticiones por periodo. Las muestras ( $1.8 \mathrm{~g}$ ) en bolsas de dacrón fueron colocadas en el primer compartimiento digestivo de una alpaca fistulada empleándose seis tiempos de digestión $(6,12$, 24, 48, 72 y 96 h). En la cinética de digestión se evaluó la digestibilidad inicial, fracción degradable, tasa de degradación, digestión potencial y tiempo medio. La digestibilidad in situ de la materia seca (DISMS) no mejoró, encontrándose diferencias estadísticas $(\mathrm{p}<0.05)$ a las 6,12 y 48 horas pero sin significancia biológica. Tampoco se encontraron diferencias para las variables en estudio. Se concluye que las enzimas fibrolíticas comerciales en la dosis utilizada no mejoran la digestibilidad ni la cinética de digestión del cogollo de caña de azúcar.

Palabras clave: enzima fibrolítica, cogollo de caña, digestibilidad in situ, alpaca

\footnotetext{
${ }^{1}$ Laboratorio de Bioquímica, Nutrición y Alimentación Animal, Facultad de Medicina Veterinaria, Universidad Nacional Mayor de San Marcos, Lima

${ }^{2}$ E-mail:fsanmatinh47@gmail.com
}

Recibido: 8 de noviembre de 2012

Aceptado para publicación: 10 de febrero de 2014 
The effect of two commercial enzymes (Allzyme Vegpro® and Rovabio ${ }^{\mathrm{TM}}$ Excel) on the in situ digestibility and kinetics of the dry matter of sugarcane bud (Saccharum officinarum) was evaluated. The substrate was dried, grounded, passed through a $3 \mathrm{~mm}$ sieve, and incubated with the enzymes for 30 minutes. The concentration of the enzymes was $1 \%$ of the substrate. In addition, a substrate without treatment was considered as control treatment. A randomized experimental design, with four periods, three treatments and two replicates per period was used. The samples $(1.8 \mathrm{~g})$ in dracon bags were placed in the first stomach compartment of one fistulated alpaca and six digestion times $(6,12$, 24, 48, 72 and $96 \mathrm{~h}$ ) were applied. The parameters evaluated in digestion kinetics were initial digestibility, degradable fraction, degradation rate, potential digestion and average time. The dry matter (ISDMD) in situ digestibility did not improve; however statistical differences $(\mathrm{p}<0.05)$ were found at 6,12 and 48 hours but without biological significance. There were no differences among treatments for the studied variables. It is concluded that the dose used of fibrolytic enzymes does not increase digestibility or kinetics of digestion of sugarcane bud.

Key words: fibrolytic enzyme, sugarcane bud, in situ digestibility, alpaca

\section{INTRODUCCIÓN}

Se estima que para el año 2025 la población humana se incrementará en un $60 \%$ (Delgado et al., 1999), de allí que la producción de alimentos de origen animal enfrenta un importante desafío para sopesar la mayor demanda. Una de las alternativas para enfrentar esta demanda es el aumento de los niveles de producción de los rumiantes, teniendo en cuenta la capacidad de estos animales de producir alimentos de alto valor biológico a partir de alimentos de baja concentración de nutrientes y no utilizables en otras especies (Delgado et al., 1999).

Los recursos forrajeros juegan un papel fundamental en la nutrición de rumiantes. Estos animales han desarrollado una relación benéfica mutua con sus microorganismos y enzimas para utilizar los carbohidratos presentes en la pared celular de los forrajes (Wilkins, 2000). El mayor obstáculo para la degradación eficiente de la pared celular en el rumen lo constituyen los cruces entrelaza- dos entre carbohidratos estructurales y otros compuestos que limitan el acceso de las enzimas a los sustratos (Mc Donald et al., 2010).

El uso de enzimas fibrolíticas para dietas de rumiantes tiene un atractivo interés, debido a las respuestas positivas en digestibilidad del forraje y la respuesta animal. La aplicación de enzimas en forrajes previamente a la incubación in vitro mejora la digestión de materia seca y fibra detergente neutro (Gado et al., 2009) sugiriendo que la aplicación de enzimas fibrolíticas momentos antes de la alimentación del ganado puede incrementar la digestión del forraje. Sin embargo, el uso de estas enzimas en dietas de rumiantes ha tenido resultados contradictorios. Algunos estudios demuestran un incremento en la producción láctea en el bovino (Yang et al., 2000), mientras que en otros no se producen tales incrementos (Sutton et al., 2003); asimismo, estudios de digestibilidad muestran tanto un efecto positivo de este parámetro (Yang et al., 2000) como nulo (Lewis et al., 1999). 
El modo de acción de estas enzimas en rumiantes no ha sido claramente identificado y estas discrepancias pueden ser debido a diferencias en la actividad enzimática, rango de aplicación y composición, estado fisiológico de los animales, modo de aplicación de la enzima, actividad ruminal y estabilidad de las enzimas, especificidad de las enzimas y la porción de la dieta que la enzima es aplicada (Beauchemin et al., 2004; Adesogan, 2005). En la nutrición animal existe una gran disponibilidad de productos enzimáticos que son comúnmente usados en monogástricos y se caracterizan por ser mezclas con diferentes características (Vahjen y Simon, 1999).

Por lo señalado, se planteó el presente estudio con el objetivo de evaluar el efecto de dos complejos enzimáticos comerciales sobre la digestibilidad in situ y la cinética de digestión de un producto forrajero de amplia disponibilidad, el cogollo de caña de azúcar (Saccharum officinarum), que no es aprovechado por su elevado y complejo contenido fibroso.

\section{Materiales y Métodos}

\section{Lugar de Ejecución}

El presente estudio se realizó a cabo en el Laboratorio de Bioquímica, Nutrición y Alimentación Animal de la Facultad de Medicina Veterinaria de la Universidad Nacional Mayor de San Marcos, Lima.

Se utilizó una alpaca macho adulta de la raza Huacaya de seis años de edad, peso aproximado de $60 \mathrm{~kg}$, y fistulada a nivel del primer compartimiento estomacal. El animal se mantuvo en un corral de $8 \times 6 \times 1.5 \mathrm{~m}$, con bebedero y comedero de cemento y la dieta fue a base de cogollo de caña y heno de alfalfa ofrecido ad libitum.

\section{Tratamientos}

Se utilizaron dos complejos enzimáticos comerciales: Allzyme Vegpro® (T1) y
Rovabio $^{\mathrm{TM}}$ Excel (T2). Estos compuestos, presentados en estado sólido, contienen celulasas, xilanasas, pectinasas, proteasas y $\beta$-glucanasas.

Se utilizó como sustrato muestras de cogollo de caña de azúcar (Saccharum officinarum) secada a $65^{\circ} \mathrm{C}$ por $48 \mathrm{~h}$, molida y pasada por un tamiz de $3 \mathrm{~mm}$. El sustrato fue tratado con las enzimas por $30 \mathrm{~min}$ antes de la incubación, en una proporción del 1\%, lo que es equivalente a 10 veces la especificación de los fabricantes cuando se usa en alimentos de aves y cerdos. Adicionalmente, se tuvo un sustrato sin ser tratado y que fue considerado como tratamiento control (T0).

El diseño experimental fue de bloques al azar, con cuatro periodos, tres tratamientos (T0, T1 y T2) y dos repeticiones por periodo-tratamiento.

\section{Digestibilidad in situ}

La digestibilidad in situ (DIS) se estimó de acuerdo al procedimiento descrito por San Martín et al. (1984), empleando bolsas de dacrón de 8.0 x $5.5 \mathrm{~cm}$, con doble costura de hilo nylon invisible y sin formación de ángulos en las esquinas. Las bolsas previas a su uso fueron lavadas, identificadas y secadas a $65^{\circ} \mathrm{C}$ por $48 \mathrm{~h}$, enfriadas en un desecador y pesadas.

Se colocó $1.8 \mathrm{~g}$ de muestra en cada bolsa, atadas en forma de bolsa de tabaco a un cordón de $80 \mathrm{~cm}$ de largo. Las bolsas fueron introducidas en el primer compartimiento estomacal de la alpaca fistulada, guardando una distancia de $10 \mathrm{~cm}$ entre bolsas y manteniendo una distancia de $20 \mathrm{~cm}$ entre la cánula y la primera bolsa. Las bolsas fueron atadas a la cánula.

Los tiempos de incubación fueron de 0 , $6,12,24,48,72$ y 96 horas. Cada tratamiento constó de dos repeticiones por periodo, cada repetición con siete tiempos y cada tiempo con una muestra, dando un total de 14 bolsas por tratamiento y por periodo. De estas, 12 
bolsas por tratamiento fueron colocadas en el animal fistulado.

Para la estimación de la digestibilidad de la materia seca para cada tiempo y tratamiento se utilizó la siguiente fórmula:

$$
\text { DESM }=100-\left[\frac{(A-B) \times 100}{C}\right]
$$

donde DISMS: Digestibilidad in situ de materia seca; A: peso de bolsa más residuo no digerido; B: peso de la bolsa; y C: peso de la materia seca inicial.

\section{Cinética de Digestión}

Para describir la cinética de digestión de la materia seca se utilizó el modelo de Orskov y McDonald (1979), según la fórmula $\mathrm{Y}=\mathrm{a}+\mathrm{b}\left(1-\mathrm{e}^{-\mathrm{ct}}\right)$, donde $\mathrm{Y}$ : Digestibilidad de la materia seca en un tiempo t; a: Intercepto de la degradación cuando $\mathrm{t}=0$ (degradabilidad inicial), \%, fracción soluble; b: Fracción insoluble potencialmente digerible, \%; c: Tasa de degradación de b; t: Tiempo de incubación, $\mathrm{h}$.

Los parámetros evaluados fueron:

- Digestibilidad inicial (a): Representa el sustrato soluble y completamente digestible que sale rápidamente de la bolsa.

- Fracción degradable (b): Es la fracción insoluble potencialmente degradable por acción fermentativa de los microorganismos del primer compartimiento estomacal.

- Tasa de degradación (c): Es la cantidad de sustrato que puede ser degradada por unidad de tiempo. Es estimada a partir del parámetro «c» del modelo anteriormente definido. Tasa de degradación de la fracción $\mathrm{b}$.

- Digestión potencial $(a+b)$ : Es definida como la degradación máxima que sufre el alimento en el ecosistema del primer compartimento si es que las condiciones presentes y el tiempo de retención en dicho ecosistema no son limitantes. Estimada a partir de la suma de los parámetros «a»y «b» del modelo.
- Tiempo medio, $h(T 1 / 2)$ : Es el tiempo necesario para que se degrade la mitad del alimento potencialmente degradable contenido en la bolsa. Este parámetro es derivado del modelo que describe la cinética de digestión: $\mathrm{T}^{1 / 2}, \mathrm{~h}=0.693 / \mathrm{c}$, donde $\mathrm{c}$ es la tasa de degradación de b.

\section{Análisis Químico Nutricional}

Se estimó el contenido de materia seca, ceniza, extracto etéreo y proteína cruda del cogollo de la caña de azúcar de acuerdo a los procedimientos AOAC (1990). La fibra cruda fue determinada según los procedimientos descritos por Van Soest et al. (1991).

\section{Análisis de la Información}

Las variables digestibilidad, digestibilidad inicial, fracción degradable, tasa de degradación, digestión potencial y tiempo medio fueron analizadas mediante análisis de varianza de dos vías. Para la diferencia múltiple de medias se utilizó la prueba de Tukey usando el PROC NLIN de SAS/STAT® v. 9.2 (SAS Institute, 2010).

\section{Resultados y Discusión}

Los resultados del análisis proximal del cogollo de caña se presentan en el Cuadro 1. Los valores son similares a reportes de la literatura para proteína (López et al., 2003; Suárez et al., 2011) y fibra cruda (López et al., 2004).

El efecto de las enzimas fibrolíticas sobre el cogollo de caña, en líneas generales, no mejora la DISMS, encontrándose diferencias significativas a favor del tratamiento control (T0) a las 6, 12 y 48 horas de incubación, pero de escasa significancia biológica. Asimismo, los parámetros de la cinética de digestión no muestran diferencias entre tratamientos con excepción de la fracción degradable (b) (Cuadro 3), aunque esta diferencia tampoco es biológicamente importante. 
Cuadro 1. Análisis proximal del cogollo de caña de azúcar (\% base seca)

\begin{tabular}{lr}
\hline Componente & Contenido (\%) \\
\hline Materia seca & 34.9 \\
Proteína & 4.48 \\
Fibra cruda & 29.1 \\
Extracto libre de N & 58.2 \\
Extracto etéreo & 1.78 \\
Cenizas & 6.44 \\
\hline
\end{tabular}

Otros estudios han demostrado la ausencia de un efecto significativo de las enzimas fibrolíticas sobre la DISMS utilizando diversos sustratos, como rastrojo de maíz y paja de avena (Yescas et al., 2004), caña de azúcar integral (Cano et al., 2003), heno del alfalfa a diferentes edades (Pinos et al., 2005) y heno de trigo (Pollard et al., 2001). Sin embargo, efectos positivos han sido reportados por otros autores con alfalfa (Pollard et al., 2001; Flores et al., 2006), aunque a veces requiriendo una alta concentración de la enzima (Pinos et al., 2001).

Cuadro 2. Digestibilidad in situ de la materia seca del cogollo de caña de azúcar tratada con enzimas fibrolíticas en función al tiempo de incubación

\begin{tabular}{ccccccc}
\hline \multirow{2}{*}{ Tratamiento $^{1}$} & \multicolumn{5}{c}{ Tiempo de incubación (h) } \\
\cline { 2 - 6 } & 6 & 12 & 24 & 48 & 72 & 96 \\
\hline T0 & $22.41^{\mathrm{a}}$ & $30.43^{\mathrm{a}}$ & $40.50^{\mathrm{a}}$ & $48.30^{\mathrm{a}}$ & $52.53^{\mathrm{a}}$ & $55.08^{\mathrm{a}}$ \\
T1 & $20.59^{\mathrm{b}}$ & $28.14^{\mathrm{b}}$ & $39.75^{\mathrm{a}}$ & $47.69^{\mathrm{ab}}$ & $52.36^{\mathrm{a}}$ & $55.06^{\mathrm{a}}$ \\
T2 & $22.05^{\mathrm{a}}$ & $29.07^{\mathrm{ab}}$ & $39.88^{\mathrm{a}}$ & $47.04^{\mathrm{b}}$ & $52.13^{\mathrm{a}}$ & $54.92^{\mathrm{a}}$ \\
\hline
\end{tabular}

${ }^{1}$ T0: Sin enzima; T1: Enzima Allzyme Vegpro®; T2: Enzima Rovabio ${ }^{\mathrm{TM}}$ Excel

$a, b$ Superíndices diferentes dentro de columnas indican diferencia estadística $(p<0.05)$

Cuadro 3. Parámetros de la cinética de digestión de la materia seca del cogollo de caña de azúcar tratada con enzimas fibrolíticas

\begin{tabular}{lccc}
\hline \multirow{2}{*}{ Parámetros } & \multicolumn{3}{c}{ Tratamientos } \\
\cline { 2 - 4 } & T0 & T1 & T2 \\
\hline Digestibilidad inicial (a) (\%) & $12.84^{\mathrm{a}}$ & $11.88^{\mathrm{a}}$ & $12.99^{\mathrm{a}}$ \\
Fracción degradable (b) (\%) & $41.94^{\mathrm{a}}$ & $43.42^{\mathrm{b}}$ & $41.83^{\mathrm{a}}$ \\
Tasa de degradación (c) & $0.044^{\mathrm{a}}$ & $0.040^{\mathrm{a}}$ & $0.040^{\mathrm{a}}$ \\
Digestión potencial (a+b) (\%) & $54.78^{\mathrm{a}}$ & $55.30^{\mathrm{a}}$ & $54.82^{\mathrm{a}}$ \\
Tiempo medio, horas & $15.94^{\mathrm{a}}$ & $17.46^{\mathrm{a}}$ & $17.38^{\mathrm{a}}$ \\
\hline
\end{tabular}

${ }^{1}$ T0: Sin enzima; T1: Enzima Allzyme Vegpro®; T2: Enzima Rovabio ${ }^{\text {TM }}$ Excel

$a, b$ Superíndices diferentes dentro de filas indican diferencia estadística $(p<0.05)$ 
Moreno et al. (2007) encontraron que la enzima Fibrozyme, en dosis de $2 \mathrm{~g} / \mathrm{kg}$, mejoró la DIVMS de dietas para vacas lecheras en las 12 primeras horas de incubación, no observándose ningún efecto después de este tiempo, indicando que la viabilidad de la enzima dependería del tiempo de exposición al medio ambiente ruminal. Estos resultados apoyan la hipótesis que las enzimas incrementan la tasa de degradación de los alimentos pero no la extensión de la misma (Giraldo et al., 2007).

Se ha demostrado que no todas las enzimas resultan igualmente efectivas en la digestión de diferentes sustratos, por lo que la actividad de un mismo preparado parece variar significativamente cuando el sustrato cambia (Zobell et al., 2000). Asimismo, en el trabajo de Pinos (1999), con seis niveles de enzimas fibrolíticas ( 0 a $80 \mathrm{mg}$ enzima por $100 \mathrm{mg}$ de sustrato), se demostró que la degradación in vitro de la fibra detergente neutro de heno de alfalfa se incrementó linealmente con la dosis sin llegar al punto de saturación, lo cual evidencia que el nivel de enzimas es dependiente del sustrato (Beauchemin et al., 1995).

\section{ConClusiones}

Las enzimas fibrolíticas comerciales Allzyme Vegpro® y Rovabio ${ }^{\mathrm{TM}}$ Excel en dosis de $1 \%$ no mejoran la digestibilidad ni la cinética de digestión de la materia seca del cogollo de caña de azúcar (Saccharum officinarum).

\section{Literatura Citada}

1. Adesogan A. 2005. Improving forage quality and animal performance with fibrolytic enzymes. Proc $16^{\text {th }}$ Florida Ruminant Nutrition Symposium. University of Florida, Gainesville. p 91109.
2. AOAC. 1990. Official methods of analysis of the Association of Official Analytical Chemists. Vol $1.15^{\text {th }} \mathrm{ed}$. Washington DC: AOAC. 1298 p.

3. Avellaneda J, Gonzales S, Pinos J, Hernández A, Montañez O, Ayala J. 2003. Enzimas fibrolíticas exógenas en la digestibilidad in vitro de cinco ecotipos de Brachiaria. Agronomia Mesoamericana 18(1): 11-17.

4. Beauchemin A, Rode M, Karren D. 1999. Use of feed enzymes in feedlot finishing diets. Can J Anim Sci 79: 243246.

5. Beauchemin K, Colombatto D, Morgavi D. 2004. A rationale for the development of feed enzyme products for ruminants. Can J Anim Sci 84: 23-36.

6. Beauchemin K, Rode L, Sewalt V. 1995. Fibrolytic enzymes increase fiber digestibility and growth rate of steers fed dry forages. J Anim Sci 75: 641-644.

7. Cano L, Aranda E, Mendosa G, Pérez J, Ramos J. 2003. Comportamiento de toretes en pastos tropicales suplementados con caña de azúcar y enzimas fibrolíticas. Téc Pec Méx 41: 153-164.

8. Delgado C, Rosegrant M, Steinfeld H, Ehui S, Courbois C. 1999. Livestock 2020. The next food revolution. Food Agriculture and the Environment Discussion Paper 28. Washington: International Food Policy Research Institute. $72 \mathrm{p}$.

9. Flores M, Ruiz F, Guerrero M, Romano J. 2006. Respuesta productiva de becerros Holstein alimentados con alfalfa de diferente calidad y enzimas fibrolíticas en la etapa pre y pos destete. Téc Pec Méx 44: 313-328.

10. Gado H, Salem A, Robinson P, Hassan M. 2009. Influence of exogenous enzymes on nutrient digestibility, extent of ruminal fermentation as well as milk production and composition in dairy cows. Anim Feed Sci Tech 154: 36-46.

11. Giraldo LA, Tejido ML, Ranilla MJ, Carro MD. 2007. Effects of exogenous fibrolytic enzymes on in vitro ruminal fermentation of substrates with different 
forage:concentrate ratios. Anim Feed Sci Tech 141: 306-321.

12. Kung L, Treacher J, Nauman A, Smagala M, Endres M, Cohen A. 2000. The effect of treating forages with fibrolytic enzymes on its nutritive value and lactation performance of dairy cows. J Dairy Sci 83: 115-122.

13. Lewis G, Sanchez W, Hunt C, Guy M, Pritchard G, Swanson B, Treacher R. 1999. Effect of direct-fed fibrolytic enzymes on the lactational performance of dairy cows. J Dairy Sci 82: 611-617.

14. López I, Aranda EM, Ramos JA, Mendoza GD. 2003. Evaluación nutricional de ocho variedades de caña de azúcar con potencial forrajero. Rev Cub Cienc Agr 37: 381-386.

15. López Y, Ramírez J, Nieves K, Fonseca F. 2004. Valor nutritivo de variedades de caña de azúcar para forraje. Pastos y Forrajes 27: 273-278.

16. McDonald P, Edwards R, Greenhalgh $J$, Morgan C, Sinclair L, Wilkinson R. 2010. Animal nutrition. $7^{\text {th }}$ ed. London: Pearson. 692 p.

17. Moreno R, Pinos JM, Gonzales S, Álvarez G, García JC, Mendoza G, Bárcena R. 2007. Efecto de enzimas fibrolíticas exógenas en la degradación ruminal in vitro de dietas para vacas lecheras. Interciencia 32: 850-853.

18. Orskov E, McDonald I. 1979. The estimation of protein degradability in the rumen from incubation measurements weighted according to rate of passage. J Agric Sci 92: 499-503.

19. Pinos J. 1999. Caracterización de enzimas fibrolíticas exógenas en la fermentación ruminal y digestibilidad de alfalfa y bacillo. Tesis de Maestría: Montecillo, México: Colegio de Postgraduados. $97 \mathrm{p}$.

20. Pinos J, González S, Mendoza G, Bárcena R, Cobos M. 2001. Efecto de enzimas fibrolíticas glucosiladas en la digestibilidad in vitro de MS y MO de alfalfa (Medicago sativa) y ballico (Lolium perenne). Rev FCV-LUZ 11: 505-509.

21. Pinos J, González S, Mendoza G, García J, Miranda L, Adriana G, Lerma V. 2005. Efecto de enzimas fibrolíticas exógenas en la degradación in vitro de ingredientes alimenticios, $\mathrm{y}$ en la producción de leche de vacas Holstein. Interciencia 30: 752-757.

22. Pollard G, Wright W, Brambl T, Richardson C, Cobb C. 2001. Effects of liquid feed supplementation and (or) cellulolytic enzymes on dry matter disappearance of either legume or grass hay. J Dairy Sci 84 (suppl 1): 37 (Abstr).

23. San Martín F, Rosales A, Valdivia R. 1984. Tasas de digestión y digestibilidad del forraje en alpaca y vacuno. Investigación sobre pastos y forrajes de Texas Tech University en el Perú. Art. Téc. T. 229-338.

24. SAS Institute Inc. 2010. SAS/STAT ${ }^{\circledR}$ 9.2 User's Guide. Cary, NC.

25. Suárez R, Mejía J, González M, García D, Perdomo D. 2011. Evaluación de ensilajes mixtos de Saccharum officinarum y Gliricidia sepium con la utilización de aditivos. Pastos y Forrajes 34: 69-86.

26. Sutton J, Phipps R, Beever D, Humphries D, Hartnell G, Vicini J, Hard D. 2003. Effect of method of application of a fibrolytic enzyme product on digestive processes and milk production in Holstein-Friesian cows. J Dairy Sci 86: 546-556.

27. Vahjen W, Simon O. 1999. Biochemical characteristics of non starch polysaccharide hydrolyzing enzyme preparations designed as feed additives for poultry and piglet nutrition. Arch Tierernahr 52: 1-14.

28. Van Soest P, Robertson J, Lewis B. 1991. Methods for dietary fiber, neutral detergent fiber, and nonstarch polysaccharides in relation to animal nutrition. J Dairy Sci 74: 3583-3597. 
29. Wilkins $R$. 2000. Forages and their role in animal systems. In: Givens DI, et al. (eds). Forage evaluation in ruminant nutrition. UK: CAB International. p 1-14.

30. Yang W, Beauchemin $K$, Rode L. 2000. A comparison of methods of adding fibrolytic enzymes to lactating cow diets. J Dairy Sci 83: 2512-2520.

31. Yescas $R$, Bárcena $R$, Mendoza $G$, González S, Cobos M, Ortega M.
2004. Digestibilidad in situ de dietas con rastrojo de maíz o paja de avena con enzimas fibrolíticas. Agrociencia 38: 23-31.

32. Zobell D, Weidmeier R, Olson K, Treacher $R$. 2000. The effect of an exogenous enzymes treatment on production and carcass characteristics of growing and finishing steers. Anim Feed Sci Tech 87: 279-285. 